\title{
Event-Related Potential Effects Associated with Insight Problem Solving in a Chinese Logogriph Task
}

\author{
Qiang Xing ${ }^{1 *}$, John X. Zhang ${ }^{2}$, Zhonglu Zhang ${ }^{1}$ \\ ${ }^{1}$ Department of Psychology, Guangzhou University, Guangzhou, China \\ ${ }^{2}$ Department of Psychology, The Chinese University of Hong Kong, Hong Kong, China \\ Email: *qiang_xingpsy@126.com
}

Received September $30^{\text {th }}, 2011$; revised November $5^{\text {th }}, 2011$; accepted December $7^{\text {th }}, 2011$

\begin{abstract}
The electrophysiological correlates of insight and non-insight problems solving were studied using eventrelated potentials (ERPs). Participants were given some time to guess Chinese logogriphs and then presented with an answer to judge whether it matched the logogriph. Results showed that the insight trials elicited a more negative ERP deflection (N300-500) than did the non-insight trials in most scalp regions. In a later time window from $600 \mathrm{~ms}$ to $1100 \mathrm{~ms}$, the insight trials elicited a more positive ERP deflection (P600-1100) than the non-insight trials, mostly in central regions. The results indicate that the early N300-500 effect may reflect cognitive conflict resulting from the breaking of mental set and the later P600-1100 effect may be related to the formation of novel associations, both crucial to the occurrence of insight.
\end{abstract}

Keywords: N300-500; P600-1100; Insight; Event-Related Potentials; Chinese Logogriph

\section{Introduction}

Early Gestalt psychologists coined the term "insight" to refer to the observation that the process of problem solving was not trial-and-error but sudden understanding of the gestalt combination of the problem elements. Insight has been generally characterized with the following features, 1) Problem solvers usually meet with a primary impasse in their first attempt to solve the problem, 2) The process of insight problem solving is non-verbal, 3) The occurrence of insight is momentary with a strong "aha" experience (Beeman et al., 2004).

Studies about insight were mostly performed with behavioral paradigms until the beginning of the 21 st century when researchers started to examine the neural mechanism of insight with brain imaging techniques such as functional magnetic resonance imaging (fMRI) and event-related potentials (ERPs). Luo et al. (Luo, 2004) for the first time studied insight with fMRI and observed activation in a number of regions during insight problem solving, including frontal cortex, temporal cortex, anterior cingulate cortex (ACC) and hippocampus. They suggested that the breaking of a mental set, crucial to the occurrence of insight, depends on ACC and left lateral prefrontal cortex, and that hippocampus plays important role in the forming of novel associations (Luo \& Niki, 2003; Luo, Niki, \& Phillips, 2004). Bowden et al. found stronger activity in anterior superior temporal gyrus (aSTG) when individuals solved insight problems compared with non-insight problems (Bowden and Beeman, 2003 \& 2007; Beeman, et al., 2004). Another study involving similar contrasts revealed increased activity in precuneus, left/ middle frontal gyrus, occipital gyrus and cerebellum (Qiu, et al., 2010). Apparently, insight involves more than a single region (Luo, 2004). Research with ERPs has also been conducted to reveal the electrophysiological substrates of insight (Qiu, et al., 2008; Wang, et al., 2009).

${ }^{*}$ Corresponding author.
Using the same catalyzed paradigm of Luo and NiKi (2003), Mai et al. (2004) asked participants to guess a Chinese logogriph for some time before the correct answer was presented. The ERP difference wave between the insight condition and the non-insight condition revealed a negative component (N380) with ACC as its neuro-generator, interpreted to reflect the breaking of mental set. Using the same paradigm, Qiu et al. (2006) found a similar response called N320, also localized in ACC. However, this N320 effect was found not only for the insight condition but also for the condition where the answer was not comprehenable. They suggested that N380, or N320 may not reflect the breaking of mental set but just a generic cognitive conflict the leve of which differs between familiar and new ways of insight problem solving (Qiu et al., 2006).

This brief review indicates that insight involves complex cognitive processes as reflected in activation in multiple regions of brain (Luo, 2004; Qiu et al., 2008). For the electrophysiological substrates of insight, there has been only one component (N380 or N320) observed that may reflect the process of breaking mental set or cognitive conflict (Mai et al., 2004; Qiu et al., 2006). Although it is indispensable to break the mental set in order to reach insight, the formation of novel association may be crucial to insight as well (Bowden \& Beeman, 2003 \& 2007; Beeman, et al., 2004; Luo, 2004; Luo \& Niki, 2003; Luo, Niki, \& Phillips, 2004), as insight is one form of creativity. So far no study has looked at how novel association is reflected in the ERPs. One possibility is that there are mulitple ERP responses under the catalyzed paradigm associated with breaking mental set and new association formation. Thus, it is hypothesized that an ERP response similar to N380 or N320 will be elicited, which may reflect breaking mental set, later, some more ERP effects would be observed which may be related to new association formation.

In real life individuals often solve an insight problem at the help of hints after a long time of exhaustive thinking, as de- 
scribed in the four-stage model of Wallas (1926). We intended to use ERP to monitor this process of insight problem solving by using the Chinese logogriphs, one of the typical insight materials. Early studies (Luo, 2004; Mai et al., 2004; Qiu et al., 2006; Qiu et al., 2008; Wang et al., 2009; Qiu et al., 2010) indicate that Chinese logogriphs are difficult because they contain misleading information. Once the answer has been guessed, individuals would feel a sudden "aha" experience.

\section{Methods}

\section{Participants}

As paid volunteers, 12 healthy undergraduates who were all native Chinese speakers ( 6 females) aged 22 years - 24 years (mean age $=23.4$ years) participated in the experiment. They are in the second year with the major of psychology. All were right-handed with normal or corrected-to-normal vision.

\section{Stimuli}

As in Qiu et al. (2008), 150 pairs of hint logogriphs and target logogriphs were used as the materials. Similar to Qiu et al. (2008) and Wang et al. (2009), the length of most logogriphs was between 2 and 6 Chinese characters, while all answers were a single character. The words that appeared in both the questions and the answers were of high frequency. The characters were presented in the Song Ti font, at size No. 16. The hint logogriphs were helpful for the guessing of target ones. For example, the hint logogriph “有口难言 (meaning difficult to say even having a mouth)" and the answer "哑 (meaning deaf)" was paired with the target logogriph “有眼难见 (meaning difficult to see even having eyes)" and the answer "盲 (meaning blind)" (for more details see (Qiu et al., 2008).

\section{Procedure}

There were 4 phases in the experiment (shown in Figure 1). Firstly, subjects were asked to try guessing the target logogriphs for $6 \mathrm{~s}$. If they got an answer, they shall press the "1" key to enter the fourth phase; if not, they shall not press any key. After a $1 \mathrm{~s}$ interval, both the hint logogriph and the answer were presented in the center for $4.5 \mathrm{~s}$, and subjects were asked to understand the relation between the hint logogriph and their answer. They shall press the "1" key if they understood the relation, and not press any key if they did not. The target logogriph was presented in the third phase for $6 \mathrm{~s}$ after a $1 \mathrm{~s}$ interval, if subjects guessed the logogriphs, they were asked to press the "1" key but to press no key if they did not. Finally, after $1 \mathrm{~s}$ interval, pairs of the target logogriph and the answer were presented for $4 \mathrm{~s}$, and subjects were asked to judge whether what their guess was consistent with the correct answer or not, and to press the "1" key if they got the answer right, or the " 2 " key if they did not but understood the relation between the logogriphs and the answers. They shall not press any key if they neither guessed the logogriph nor understood the correct answer. During the last phase, the correctly guessed condition was regarded as the non-insight condition. The condition where subjects understood the answer was referred to as the insight condition according to early studies (Mai et al., 2004; Qiu et al., 2006). In the non-insight condition, the feedback answer was consistent with what participants guessed, insight did not occur when they saw the answers, in contrast, in the insight condition, participants did not guess out the answers or had incorrect answers, once they understood the standard answers after their presentation where an "aha" experience occurred (Mai et al., 2004).

To be familiar with the procedure and pace of the task, subjects were trained with 10 trials using 10 pairs of practice logogriphs in the same procedure. The 150 pairs of test logogriphs were evenly divided into 5 blocks with each pair of stimuli presented randomly without any repetition. Subjects could take short breaks between two blocks. Subjects were seated in a quiet room with the eyes being $70 \mathrm{~cm}$ away from the screen. They were instructed to respond as quickly and accurately as possible but avoid movements and blinks.

\section{ERP Recording}

Brain electrical activities were recorded from 32 scalp sites using tin electrodes mounted in an elastic cap (Brain Product) with the reference located between the Fz electrode and the $\mathrm{Cz}$ electrode. The vertical electrooculogram (EOG) was recorded with one electrode placed above the right eye and the horizontal EOG was recorded with the other electrode placed left at the left eye. All the interelectrode impedance was maintained below $5 \mathrm{k} \Omega$. The EEG and EOG were amplified using a 0.05-80 $\mathrm{Hz}$ bandpass filter, continuously sampled at $500 \mathrm{~Hz} /$ channel for off-line analysis. Eye movement artifacts including blinks and eye movements were rejected off-line. High frequency noise was removed by applying a low-pass filter set at $16 \mathrm{~Hz}$. Before average, trials contaminated by blinks, eye movements and excessive muscle activity (voltage over \pm 80 uv in any channel) were rejected off-line. During averaging, all scalp-recorded activity was digitally re-referenced to an average of the left and right mastoids. ERPs following the onset of the answers (together with the target logogriphs) were analyzed within 1100 $\mathrm{ms}$ setting the pre-stimulus $200 \mathrm{~ms}$ period as the baseline. EEGs of the correct guessing condition (the non-insight condition) and the understanding condition (the insight condition) were averaged separately.

\section{ERP Analysis}

As observed from the grand-averaged waveform and topographical maps (Figure 2 and Figure 3), ERPs elicited by the answer stimuli for the insight and the non-insight conditions

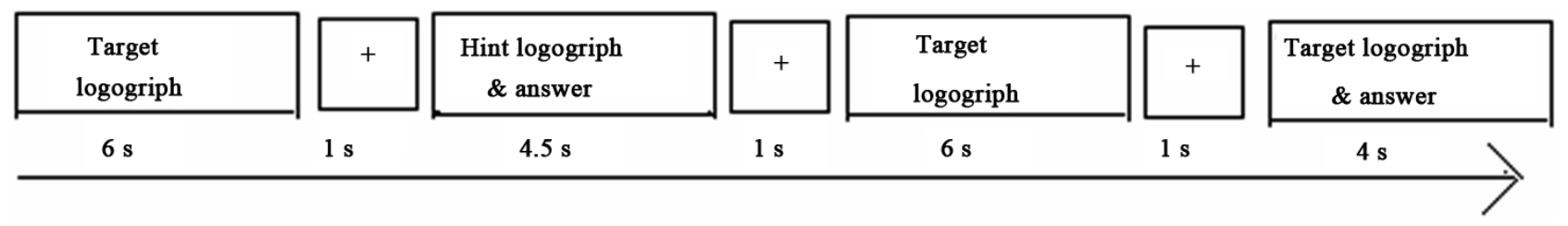

Figure 1.

The flow of guessing logogriph procedure in each trial. 
were clearly different from each other. The difference waves were obtained by subtracting the averaged ERP of the noninsight condition from that of the insight condition. Mean amplitudes in the time windows of $300 \mathrm{~ms}-500 \mathrm{~ms}$ and $600 \mathrm{~ms}$ $1100 \mathrm{~ms}$ were measured based on inspection of the grand-averaged waveform and the topographical maps.

A negative ERP deflection (N300-500) was evoked in the time window between $300 \mathrm{~ms}$ and $500 \mathrm{~ms}$. Based in visual inspection of the results and results in early studies (Mai et al., 2004; Qiu et al., 2006), the following 13 electrodes were chosen for two-way repeated-measures analyses of variance (ANOVA). The ANOVA factors were response type (insight; non-insight) and brain scalp region [frontal (F3, F4, Fz), central (C3, $\mathrm{C} 4, \mathrm{Cz})$, parietal $(\mathrm{P} 3, \mathrm{P} 4, \mathrm{Pz})$, fronto-central $(\mathrm{FC} 1, \mathrm{FC} 2)$ and centro-parietal (CP1, CP2) (the averaged ERP amplitude of electrodes in each region was pooled)]. A positive ERP deflection (P600-1100) was elicited in the time window between 600 $\mathrm{ms}$ and $1100 \mathrm{~ms}$, mostly in frontal, fronto-central and central regions as shown in the topographical maps. So the following 8 electrodes were chosen for two-way repeated-measures analyses of variance. The ANOVA factors were response type (insight; non-insight) and brain scalp region [frontal (F3, F4, Fz), central $(\mathrm{C} 3, \mathrm{C} 4, \mathrm{Cz})$, fronto-central $(\mathrm{FC} 1, \mathrm{FC} 2)$ (pooling electrodes in each region)]. P-value of the analyses of variance was corrected using the Greenhouse-Geisser method. The statistical analyses were made in SPSS 13.0.

\section{Results}

\section{Behavioral Results}

For the non-insight condition, the average number of guessing the correct answer was $82 \pm 18$ and the reaction times were $1285 \pm 324 \mathrm{~ms}$. For the insight condition, the average number of understanding the answer was $51 \pm 17$ and the reaction times were $3097 \pm 452 \mathrm{~ms}$. The reaction times under the insight condition were significantly longer than the non-insight condition, $\mathrm{F}(1,11)=412.43, p<.001$.

\section{ERP Results}

As shown in the grand-averaged waveforms and the difference wave map (Figure 2 and Figure 3), the early ERP component (N1) was elicited under both the non-insight and insight conditions, with no main effect of response type. However, the insight condition evoked a more negative ERP deflection (N300 500) than the non-insight condition in the time window between $300 \mathrm{~ms}$ and $500 \mathrm{~ms}$ for most of the scalp regions. Later within the $600 \mathrm{~ms}-1100 \mathrm{~ms}$ window, a more positive ERP component (P600-1100) was elicited under the insight condition than the non-insight condition, salient in frontal, fronto-central and central scalp regions. The mean ERP amplitudes for the $300 \mathrm{~ms}-500 \mathrm{~ms}$ and $600 \mathrm{~ms}-1100 \mathrm{~ms}$ time windows were selected for statistical analysis below.

Two-way repeated-measures ANOVA showed that the main effect of the response type reached significance in the $300 \mathrm{~ms}$ $500 \mathrm{~ms}$ window, $\mathrm{F}(1,11)=16.51, p<.001$. The main effect of region was not significant, $\mathrm{F}(4,44)=2.67, p>.1$. The interaction effect was not significant, $\mathrm{F}<1$. Hence, the insight trials elicited a significantly more negative ERP deflection (N300500) than the non-insight trials between $300 \mathrm{~ms}$ and $500 \mathrm{~ms}$. Between $600 \mathrm{~ms}$ and $1100 \mathrm{~ms}$, there was no main effect for response type or region, $\mathrm{F}(1,11)=2.46, p>.1, \mathrm{~F}(2,22)=$
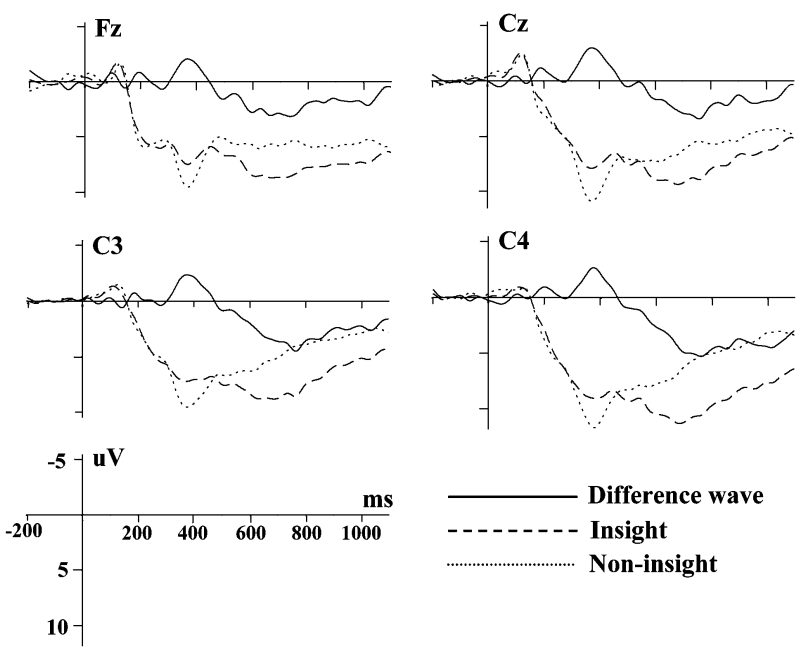

Figure 2.

Grand-averaged ERPs at Fz, Cz, C3 and C4 for the insight (long dotted lines), non-insight conditions (short dotted lines), and the difference wave (insight minus non-insight, solid lines).
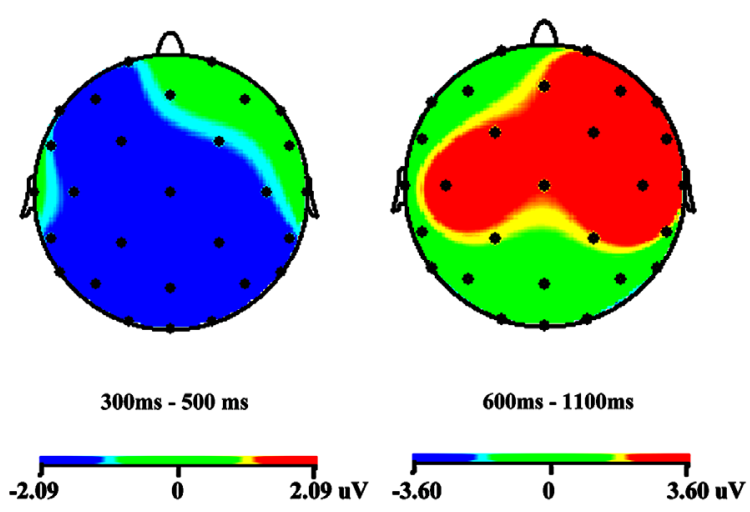

Figure 3.

Topographical maps of the voltage amplitudes for the insight vs. non-insight difference wave in the $300 \mathrm{~ms}-500 \mathrm{~ms}$ and $600 \mathrm{~ms}-$ $1100 \mathrm{~ms}$.

$2.85, p>.1$, respectively. The interaction between response type and region was significant, $\mathrm{F}(2,22)=4.47, p<.05$. Simple effect analysis showed that the insight trials elicited a more positive ERP component (P600-1100) than non-insight over the central regions, $\mathrm{F}(1,11)=5.05, p<.05$.

\section{Discussion}

Both the insight and non-insight conditions elicited an N1 showing no difference by response type. This result indicates that N1 was related to the early visual processing that was comparable across the two conditions each involving the presentation of between 3 and 7 characters.

In later time windows, consistent with early studies, the insight stimuli elicited a more negative ERP deflection (N300500 ) in the $300-500$ time window than the non-insight stimuli, similar to the N380 in Mai et al. (2004) and the N320 in Qiu et al. (2006). Difference in the latency of the three ERP components may be attributed at least partially to the differences of the logogriph length.

As described in the introduction, it remains unclear as to 
what cognitive mechanism this negative effect reflects, the breaking of mental set (Mai, et al., 2004) or cognitive conflict (Qiu, et al., 2006). In the catalyzed paradigm, subjects firstly formed certain thought (the old thought). They then formed a new thought if they understood the logogriphs or they did not form any clear thought if they did not understand the logogriphs. Either way, they would experience transition from the old thought to a new thought or to no thought at all, inducing cognitive conflict. In the present study, the focus was on the insight condition involving only comprehension, the conflict from switching from an old thought to a new thought shall by nature be related to the breaking of mental set.

Breaking mental set was regarded as one key cognitive process of insight (Qiu, et al., 2010; Zhao, et al., 2011). Insight problems such as the Chinese logogriphs task often involve misleading cues, which make inappropriate constraints or unhelpful primary knowledge strongly activated, leading to impasse where individual does not know how to solve this problem. Therefore, it is necessary to break the mental set in order to solve the insight problem. However, insight as one form of creativity involves another crucial cognitive component, the formation of novel association (Bowden and Beeman, 2003 \& 2007; Luo, 2004) as well, therefore differences between the two components lie in that the former emphasizes more the breaking of the old and non-effective association while the later more on the forming of new and effective association. Hence different ERP components were observed in the time course of insight occurence.

Beyond the early effects already reported in previous studies (Mai et al., 2004; Qiu et al., 2006), the insight condition also elicited a more positive ERP deflection (P600-1100) between $600 \mathrm{~ms}$ and $1100 \mathrm{~ms}$ in the present study, mostly in frontal, fronto-central and central regions (reaching significance in central regions). No similar effects in $\mathrm{P} 300$ or late positive component (LPC) had been observed for insight in early studies (Mai, et al., 2004; Qiu et al., 2006). P300 and LPC shared many similarities in latency and topographical distributions and were considered related (Hajcak, Moser, \& Simons, 2006; Huang \& Luo, 2009). LPC was found to be involved in attentional and orienting processes (Knight; 1996; Hajcak, Moser, \& Simons, 2006), with its amplitude reflecting the amount of mental resources employed (Olofsson, Nordin, Sequeira, \& Polich, 2008). P300 was linked to memory updating, encoding, or retrieval, and the formation of new representations through integration (Donchin, 1981), with its amplitude reflecting deployment of attentional resources (Donchin \& Coles, 1988). We suggest that when the answers appeared, there was conflict between the new and old thoughts under the insight condition, shown in the N300-500 effect. To correctly understand the answers of logogriphs, individuals needed to retrieve information related to the answer and loose constraints of the old thought. When the retrieved information was successfully integrated with the given answers forming a novel association or a new representation, the insight occurred. P300 or LPC was sensitive to this process probably because of the critical role of attentional resources in this process. In conclusion, the P600-1100 effect might reflect the forming of novel associations following the breaking of mental set.

In a word, the present ERP study showed that, compared with non-insight problem solving, insight problem solving elicited a negative deflection in the time window of $300 \mathrm{~ms}-500$ $\mathrm{ms}$ and a positive deflection between $600 \mathrm{~ms}$ and $1100 \mathrm{~ms}$. Therefore, the significance of this research is that the same result was repeated that $\mathrm{N} 300-500$ may be related to the cogni- tive conflict in the breaking of mental set, furthermore, P6001100 was discovered which may be related to the formation of novel associations. So both N300-500 and P600-1100 may be important electrophysiological labels in the process of insight problem solving. However, there may be limitation as well as significance. Similar to early studies (Mai et al., 2004; Qiu et al., 2006), the catalyzed paradigm was employed in this study, under which insight is externally elicited while internally produced insight would be neglected to some extent (Qiu et al., 2008).

\section{Acknowledgements}

This work was supported by the National Natural Science Foundation of China (31070918).

\section{REFERENCES}

Bowden, E. M., \& Jung-Beeman, M. (2003). Aha! Insight experience correlates with solution activation in the right hemisphere. Psychonomic, Bulletin and Review, 10, 730-737. doi:10.3758/BF03196539

Bowden, E. M., \& Jung-Beeman, M. (2007). Methods for investigating the neural components of insight. Methods, 42, 87-99. doi:10.1016/j.ymeth.2006.11.007

Donchin, E. (1981). Surprise! Surprise? Psychophysiology, 18, 493-513. doi:10.1111/j.1469-8986.1981.tb01815.x

Donchin, E., \& Coles, M. G. H. (1988). Is the P300 component a manifestation of context updating? Behavioral Brain Science, 11, 355 372. doi:10.1017/S0140525X00058027

Hajcak, G., Moser, J. S., \& Simons, R. F. (2006). Attending to affect: Appraisal strategies modulate the electrocortical response to arousing pictures. Emotion, 6, 517-522. doi:10.1037/1528-3542.6.3.517

Huang, Y. X., \& Luo, Y. J. (2009). Can negative stimuli always have the processing superiority? An ERP study. Acta Psychologica Sinica, 41, 822-831.

Jung-Beeman, M., Bowden, E. M., Haberman, J., Frymiare, J. L., Arambel-Liu, S., Greenblatt, R., et al. (2004). Neural activity when people solve verbal problems with insight. PLoS Biology, 2, 500-510. doi:10.1371/journal.pbio.0020097

Knight, R. T. (1996). Contribution of human hippocampal region to novelty detection. Nature, 383, 256-259. doi:10.1038/383256a0

Luo, J. (2004). Neural correlates of insight. Acta Psychologica Sinica, $36,219-234$.

Luo, J., \& Niki, K. (2003). Function of hippocampus in "insight" of problem solving. Hippocampus, 13, 316-323.

doi:10.1002/hipo.10069

Luo, J., Niki, K., \& Phillips, S. (2004). Neural correlates of the "Aha! Reaction". NeuroReport, 15, 2013-2017. doi:10.1097/00001756-200409150-00004

Mai, X.Q., Luo, J., Wu, J. H., \& Luo, Y. J. (2004). “Aha!” effects in guessing riddle task: An ERP study. Human Brain Mapping, 22, 261-270. doi: $10.1002 / \mathrm{hbm} .20030$

Olofsson, J. K., Nordin, S., Sequeira, H., \& Polich, J. (2008). Affective picture processing: An integrative review of ERP findings. Biological Psychology, 77, 247-265. doi:10.1016/j.biopsycho.2007.11.006

Qiu, J., Li, H., Jou, J. W., Liu, J., Luo, Y. J., Feng, T. Y., et al. (2010). Neural correlates of the "Aha" experiences: Evidence from an fMRI study of insight problem solving. Cortex, 46, 397-403. doi:10.1016/j.cortex.2009.06.006

Qiu, J., Li, H., Luo, Y. J., Chen, A. T., \& Zhang, Q. L. (2006). Brain mechanism of cognitive conflict in a guessing Chinese logogriph task. NeuroReport, 17, 679-682. doi:10.1097/00001756-200604240-00025

Qiu, J., Li, H., Yang, D., Luo, Y. J., Li, Y., Wu, Z. Z., et al. (2008). The neural basis of insight problem solving: An event-related potential study. Brain and Cognition, 68, 100-106. doi:10.1016/j.bandc. 2008.03 .004

Wallas, G. (1926). The art of thought. New York: Harcourt Brace Jo- 


\section{Q. XING ET AL.}

vanovich.

Wang, T., Zhang, Q. L., Li, H., Qiu, J., Tu, S., \& Yu, C. Y. (2009). The time course of Chinese riddles solving: Evidence from an ERP study. Behavioral Brain Research, 199, 278-282. doi:10.1016/j.bbr.2008.12.002
Zhao, Y. F., Tu, S., Lei, M., Qiu, J., \& Zhang, Q. L. (2011). The neural basis of breaking mental set: An event-related potential study. EXperimental Brain Research, 208, 181-187.

doi:10.1007/s00221-010-2468-z 